\title{
Glaze: A Visualization Framework for Mobile Devices
}

\author{
Roberto Sousa, Valentina Nisi, and Ian Oakley \\ Dept de Matemática e Engenharias, Universidade da Madeira, Funchal, Portugal \\ robertonssousa@gmail.com, valentina.nisi@gmail.com, ian@uma.pt
}

\begin{abstract}
The processing power of mobile devices is increasing steadily; their screen size suffers more fundamental limits. Given this contrast, we identify mobile information visualization which maximizes the effectiveness of small screen displays as a key area for future development. To achieve this, we present an overview of Glaze, a plug-in based visualization framework for mobile devices based on the information visualization reference model. We discuss two prototype visualizations implemented using Glaze and designed based on the output of concept generation interviews with users.
\end{abstract}

Keywords: Visualizations, Mobile Devices, Multimedia, Framework.

\section{Introduction}

Mobile phones are now a part of many aspects of everyday life. Modern Smartphones can not only make calls, but also play music, take and store photos, browse the Internet and send email. They are arguably on the cusp of becoming truly personal computers capable of capturing, maintaining and storing large amounts of valuable information for their users. They are also becoming increasingly able to detect information about their environment. Sensors such as cameras, accelerometers, GPS and magnetometers now appear in high end devices; past trends suggest they will migrate to lower-end handsets in the near future.

The application areas opened up by such mobile data processing and sensing capabilities are broad. Navigation has been extensively explored and many map based commercial systems exist (e.g. Nokia Maps). The research community is exploring other metaphors such as augmented reality (Narzt, et al., 2003) or non-visual directional cues (Erp et al., 2007). Other sensors are more focused on user interaction. For example, the sensing of pose to control the orientation of graphical displays (Hinckley et al., 2005), motion as input to dynamic simulations (Cho et al., 2007) and camera input to perform cursor-like navigation and selection (Rohs, 2005).

One area at the convergence of these new technologies is that of information visualization. This is of particular relevance to mobile devices because of their limited display capacity - the small screens of handheld devices provide a clear imperative to design visual information carefully and with the goal of presenting it most effectively. Although this is true of the information and content natively stored on a phone (such as message, contacts and photos), it is also relevant when considering the diverse information captured by sensors. This often relates to multi-dimensional spaces, such as maps, or other rich contextual data sets, such as the markers used in augmented 
reality. This paper identifies a need for flexible frameworks to support the development of advanced sensor-enabled visualizations on mobile devices and describes Glaze, a first attempt to produce such a framework. The remainder of this paper is structured as follows: Section 2 provides an overview of Glaze; Section 3 introduces particular scenarios and visualizations that we have implemented; and Section 4 contains conclusions and speculations on the future of this work.

\section{Glaze Framework}

Existing mobile visualization frameworks typically address specific problems such as viewing networks (Kim et al., 2008) or volume data (Zhou et al., 2006). Glaze on the other hand is a general mobile visualization framework intended to support a wide range of sensor inputs and output visualizations. To this end, it was kept intentionally minimalist in structure and its architecture was based on the information visualization reference model described by Card et al. (1999) and shown in Figure 1. It was developed using C++ for the Nokia N95 (running Symbian 9.2 and S60 Rel 3.1) but features an extensible structure of plug-ins to facilitate its use with different input and output capabilities or operating systems. The overall design approach was to effectively separate responsibilities to ease development and maximize the future portability of the code.

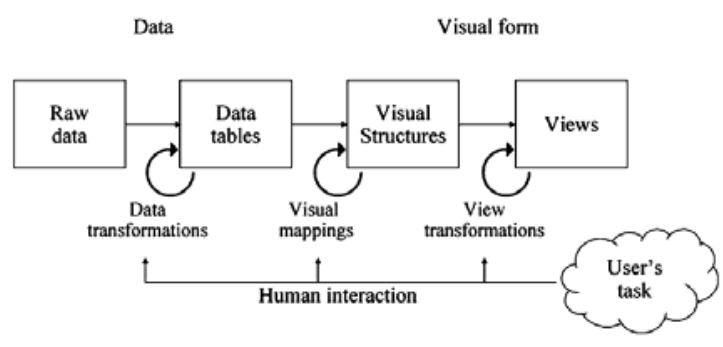

Fig. 1. Information Visualization Reference Model (from Card et al., 1999)

The framework has four components: the core, input, visualization and front-end. The core simply manages the application and implements the processes described in the information visualization reference model. The latter three components are plug-in based and can also be configured using XML, at the discretion of the developer. Plugins intended for a single purpose might offer no (or only superficial) customization options, while more sophisticated ones can introduce new schemas to control key aspects of their behavior. One immediate implication of this structure is that it allows the creation of generic visualization templates (such as fish-eyes) which can be tailored to particular data sets and scenarios defined within the XML. Components are based on the three mapping and transformation processes in Card et al.'s model and are described in more detail below.

The input component is responsible for reading and abstracting the raw data into a developer specified format suitable for further processing. Developers are able to write input plug-ins for different sensors (such as cameras, accelerometers or GPS) or files without modifying other parts of the system. 

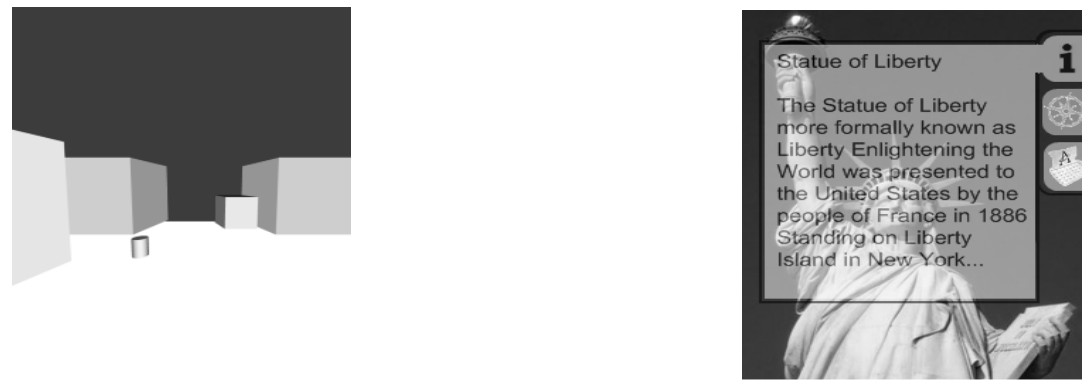

Fig. 2. Two Glaze visualizations: Nightvision (left), Information Overlay (right)

The visualization component is responsible for mapping data from input component to visual elements and specifying how these objects can be viewed and interacted with. This is a substantial task and the minimalist approach in Glaze means it is largely unconstrained: the developer of a plug-in has considerable flexibility in performing this task. The disadvantage of this approach is that it increases the complexity of constructing new visualization components. Finally, the front-end is responsible for initializing and updating the graphic side of the visualization and also of rendering the scene to the screen. It simplifies development by providing and encapsulating basic services such as device initialization and screen rendering. This explicit separation ensures that Glaze is independent from a particular rendering API; the developer can choose which API to work with by simply re-implementing the front-end. Finally, this separation also allows for visualization aggregation with API's that support a render to texture functionality. This enables the display of multiple visualizations on the screen at the same time (or the use of one visualization as the input to another) and considerably increases the expressiveness of the system. Basically, on-screen visualizations can be "mashed-up" or combined in an ad-hoc manner simply through editing the XML configuration files.

\section{Test Applications}

To gather potential uses of an advanced visualization system for mobile devices, we conducted a series of 18 semi-structured interviews with users. These involved deliberately open ended questions intended to elicit device features and applications unrelated to current technological capabilities and limitations. From this data set, we selected two candidate targets for visualization development based on their novelty, potential utility and the frequency with which they were requested. These were Night Vision and Information Overlay, both also illustrated in Figure 2.

The Night Vision system uses GPS and an external digital compass to compute the position and orientation of the user. It displays a 3D model of the area around a user mapped so that the position and orientation of the virtual scene match that of the real world. This allows users to easily see the structure and organization of their surroundings and navigate in low light environments. Information Overlay again uses the GPS, camera and an external compass to overlay relevant information about objects around 
the user on visual scene captured by the viewfinder. We have also developed several generic visualizations, such as a tagcloud and a $1 \mathrm{D}$ fish eye view.

\section{Conclusions and Future Work}

Glaze was designed to be highly general and flexible for both developers and users. It provides a minimal software structure to constrain a visualization developer's task to a systematic set of modular problems. It also provides a mechanism for end users to customize the system by combining and mixing the on-screen visualization components. This provides the ability to "mash-up" different visualizations, but also to use the output of one as the input to another. For example, a location aware map visualization could be configured as the input to a 2D fish-eye to create a richer display with a higher information density.

Future work will focus on both user evaluation and further developing the system. For example, basic input modules for the N95's camera, accelerometer and GPS have been implemented. We also plan to conduct several user studies both to provide further ideation as to novel visualizations and also to systematically evaluate those we create. In short, given the growing processing power and limited screen size of mobile devices, this paper has identified effective information visualization to be an important area for development. By introducing Glaze, a general visualization framework for handhelds, it hopes to usher in a new range of expressive, attractive and usercustomizable mobile interfaces.

\section{References}

1. Card, S.K., Mackinlay, J.D., Shneiderman, B.: Readings in information visualization: using vision to think. Morgan Kaufmann Publishers Inc., San Francisco (1999)

2. Cho, S., Choi, C., Sung, Y., Lee, K., Kim, Y., Murray-Smith, R.: Dynamics of tilt-based browsing on mobile devices. In: CHI 2007 (2007)

3. Erp, J.B., Veen, H.A., Jansen, C., Dobbins, T.: Waypoint navigation with a vibrotactile waist belt. ACM Trans. Appl. Percept. 2, 2 (2005)

4. Hinckley, K., Pierce, J., Horvitz, E., Sinclair, M.: Foreground and Background Interaction with Sensor-enhanced Mobile Devices. ACM TOCHI Special Issue on Sensor-Based Interaction, 12 (1) (2005)

5. Narzt, W., Pomberger, G., Ferscha, A., Kolb, D., Müller, R., Wieghardt, J., Hörtner, H., Lindinger, C.: Pervasive Information Acquisition for Mobile AR-Navigation Systems. In: 5th IEEE Workshop on Mobile Computing Systems \& Applications (2003)

6. Rohs, M.: Real-world interaction with camera phones. In: Murakami, H., Nakashima, H., Tokuda, H., Yasumura, M. (eds.) UCS 2004. LNCS, vol. 3598, pp. 74-89. Springer, Heidelberg (2005)

7. Kim, J., Hsu, W.-J., Moon, S., Kumar, U., Helmy, A.: Visualization and Representation of Mobile Network Users. In: 5th Annual IEEE Communications Society Conference, San Francisco, pp. 588-590 (2008)

8. Zhou, H., Qu, H., Wu, Y., Chan, M.-Y.: Volume visualization on mobile devices. In: 14th Pacific Conference on Computer Graphics and Applications, pp. 76-84. National Taiwan University Press, Taiwan (2006) 